\title{
Study of the molecular and ionized gas in a possible precursor of an ultra-compact $\mathrm{H}$ II region
}

\author{
M. E. Ortega ${ }^{1}$, S. Paron ${ }^{1,2}$, E. Giacani ${ }^{1,2}$, M. Celis Peña ${ }^{1}$, M. Rubio ${ }^{3}$, and A. Petriella ${ }^{1}$ \\ ${ }^{1}$ CONICET - Universidad de Buenos Aires, Instituto de Astronomía y Física del Espacio (IAFE), 1428 Buenos Aires, Argentina \\ e-mail: mortega@iafe.uba.ar \\ ${ }^{2}$ Universidad de Buenos Aires, Facultad de Arquitectura, Diseño y Urbanismo, Departamento de Diseño Industrial, \\ 1421 Buenos Aires, Argentina \\ 3 Departamento de Astronomía, Universidad de Chile, Casilla 36-D, Santiago, Chile
}

Received 27 March 2017 / Accepted 17 August 2017

\begin{abstract}
Aims. We aim to study the molecular and the ionized gas in a possible precursor of an ultra-compact $\mathrm{H}$ II region to contribute to the understanding of how high-mass stars build-up their masses once they have reached the zero-age main sequence.

Methods. We carried out molecular observations toward the position of the Red MSX source G052.9221-00.4892, using the Atacama Submillimeter Telescope Experiment (ASTE; Chile) in the ${ }^{12} \mathrm{CO} J=3-2,{ }^{13} \mathrm{CO} J=3-2, \mathrm{C}^{18} \mathrm{O} J=3-2$, and $\mathrm{HCO}^{+} J=4-3$ lines with an angular resolution of about 22". We also present radio continuum observations at $6 \mathrm{GHz}$ carried out with the Jansky Very Large Array (JVLA; USA) interferometer with a synthesized beam of $4 . \prime 8 \times 4$ ". 1 . The molecular data were used to study the distribution and kinematics of the molecular gas, while the radio continuum data were used to characterize the ionized gas in the region. Combining these observations with public infrared data allowed us to inquire about the nature of the source.

Results. The analysis of the molecular observations reveals the presence of a kinetic temperature and $\mathrm{H}_{2}$ column density gradients across the molecular clump in which the Red MSX source G052.9221-00.4892 is embedded, with the hotter and less dense gas in the inner region. The ${ }^{12} \mathrm{CO} J=3-2$ emission shows evidence of misaligned massive molecular outflows, with the blue lobe in positional coincidence with a jet-like feature seen at $8 \mu \mathrm{m}$. The radio continuum emission shows a slightly elongated compact radio source, with a flux density of about $0.9 \mathrm{mJy}$, in positional coincidence with the Red MSX source. The polar-like morphology of this compact radio source perfectly matches the hourglass-like morphology exhibited by the source in the $K_{\mathrm{s}}$ band. Moreover, the axes of symmetry of the radio source and the near-infrared nebula are perfectly aligned. Thus, based on the presence of molecular outflows, the slightly elongated morphology of the compact radio source matching the hourglass-like morphology of the source at the $K_{\mathrm{s}}$ band, and the lack of evidence of collimated jets in the near-infrared spectrum, one interpretation for the nature of the source, is that the Red MSX source G052.9221-00.4892 could be transiting a hyper-compact H II region phase, in which the young central star emits winds and ionizing radiation through the poles. On the other hand, according to a comparison between the $\mathrm{Br} \gamma$ intensity and the radio flux density at $6 \mathrm{GHz}$, the source would be in a more evolved evolutionary stage of an optically thin UC H II region in photoionization equilibrium. If this is the case, from the radio continuum emission, we can conjecture upon the spectral type of its exciting star which would be a B0.5V.
\end{abstract}

Key words. ISM: clouds - stars: formation - stars: winds, outflows

\section{Introduction}

The last few years have seen rapidly growing observational activity aimed at the characterization of high-mass star forming regions exhibiting a wide range of evolutionary stages that go from the "hot cores" (Cesaroni et al. 1994) to the ultra-compact (UC) H II regions (Wood \& Churchwell 1989). However, the formation of high-mass stars is not yet well understood (e.g., Zinnecker \& Yorke 2007; Tan et al. 2014), mainly because their earliest stages of evolution have typical timescales of about $10^{5} \mathrm{yr}$ (Tan \& McKee 2002). At present, two theoretical scenarios are proposed to explain the formation of these stars: a monolithic collapse of turbulent gas on the scale of massive dense cores (Tan \& McKee 2002), which is a scaled-up version of the low mass stars formation picture, and a competitive one where accretion occurs inside the gravitational potential of a clusterforming massive dense core (Bonnell \& Bate 2006).

The currently accepted evolutionary path of high-mass stars begins inside dense and massive molecular cores. The young stars finish their contraction and reach the zero age main sequence (ZAMS) very rapidly (Keto \& Wood 2006). At this point, the star begins to radiate extreme ultraviolet photons which ionize its surroundings, generating a hyper-compact (HC) H II region (Kurtz 2005). For the accretion to prevail against the radiation pressure, an accretion rate of several orders of magnitude above the typical values related to low-mass star formation is required (Garay \& Lizano 1999). However, what seems to be crucial in this issue is a non-spherical accretion, with the accreting material reaching the young star by flowing inwards, mainly through the equatorial plane (e.g., Kuiper et al. 2011). Keto (2007), based on analytical calculations, characterized different evolutionary stages of the HC H II region phase. In a first stage, arises a small quasi-spherical H II region gravitationally trapped due to the accretion flow. Then, in a second stage, the ionization increases and the HII region transitions to a bipolar morphology in which appears outward flows of ionized gas through the poles direction. Thus, the study of the HC H II regions is crucial to understanding, for example, how O-type stars acquire about the half of the final stellar mass after the star begins to produce ionizing radiation (Zhang et al. 2014). However, 
given the short lifetime of this stage and the biases toward the detection of UC H II regions of most radio continuum surveys, the number of HC H II regions cataloged is still scarce.

In this work, we report on the study of the molecular and ionized gas associated with the Red MSX source G052.9221-00.4892 (hereafter MSXG52). MSXG52 has been cataloged as an H II region in the Red MSX Source Survey (Lumsden et al. 2013), based on its associated radio continuum emission (CORNISH Survey; Hoare et al. 2012). MSXG52 is embedded in a pillar-like structure located onto the border of the infrared dust bubble MWP1G052845-005363 (hereafter bubble G52) first cataloged by Simpson et al. (2012). This irregular bubble of about 10 arcmin in size and centered at $l=$ $52.845 ; b=-0.536$, is the infrared counterpart of the H II region G052.9-00.6, which has a radio recombination line at $43.5 \mathrm{~km} \mathrm{~s}^{-1}$ (Lockman 1989). Using a flat rotation model for our Galaxy (with $R=7.6 \pm 0.3 \mathrm{kpc}$ and $\theta=214 \pm 7 \mathrm{~km} \mathrm{~s}^{-1}$ ) this velocity corresponds to the near and far distances of about $5.1 \mathrm{kpc}$ (tangent point) and $7.2 \mathrm{kpc}$, respectively. Anderson \& Bania (2009) resolved the kinematic distance ambiguity in favor of the far distance based on the HI self-absorption method. Thus, in this paper we have assumed $7.2 \mathrm{kpc}$ as the distance to the bubble G52 and MSXG52.

\section{Presentation of bubble G52, the pillar, and MSXG52}

Figure 1 shows a two-color composite Spitzer image $(24 \mu \mathrm{m}$ in red and $8 \mu \mathrm{m}$ in cyan) of bubble G52. The $24 \mu \mathrm{m}$ emission (MIPSGAL; Carey et al. 2009), which might be associated with very small grains, is confined to the interior of the bubble. The $8 \mu \mathrm{m}$ emission (GLIMPSE; Benjamin et al. 2003), which traces the photo-dissociation regions (PDRs), exhibits a semi-shell like morphology opened toward lower Galactic longitudes. The most interesting feature at this band is a structure with a conspicuous pillar morphology (indicated in the figure), which seems to have been sculpted by the action of the H II region. Figure 1a shows a magnified view of this structure in a Spitzer two-color image where the 4.5 and $8 \mu \mathrm{m}$ emissions are represented in green and red, respectively. Toward the head of the pillar, it can be appreciated a slightly elongated bright bulk of emission (seen in yellow) that corresponds to the position of MSXG52. In connection with this source, appears a curved filament that resembles a typical head-tail jet morphology pointing toward higher Galactic longitudes. Besides, the Fig. 1a shows the remarkable prominence of the $8 \mu \mathrm{m}$ emission with respect to the $4.5 \mu \mathrm{m}$ emission (which is most probably attributed to shocked $\mathrm{H}_{2}$ ) in the jet-like feature, which suggests the presence of hydrocarbons heated by FUV photons from the central source that would be illuminating cavity walls (e.g., Qiu et al. 2008; van den Ancker et al. 2000). Besides, reflection nebulae dominated by the emission from FUVheated hydrocarbons in the $8 \mu \mathrm{m}$ band may imply the presence of young B stars (Qiu et al. 2008). Figure 1b shows a magnified view of the jet-like feature and the central source seen at $8 \mu \mathrm{m}$. The bulk of the emission exhibits a slightly elongated morphology centered at the location of MSXG52 (green cross). Toward higher Galactic longitudes it extends the curved filament which connects the bulk of the emission with a bow-shock-like feature which crowns the structure. Figure 1c shows a magnified view of the central source seen at $K_{\mathrm{S}}$ band extracted of the UKIRT Infrared Deep Sky Survey (UKIDSS; Warren et al. 2007). The $8 \mu \mathrm{m}$ emission levels at 80, 100, and $140 \mathrm{MJy} \mathrm{beam}^{-1}$ are shown in the figure. The nebulosity associated with the UKIDSS source UGPS J193054.62+172842.0 (Lucas et al. 2008) exhibits
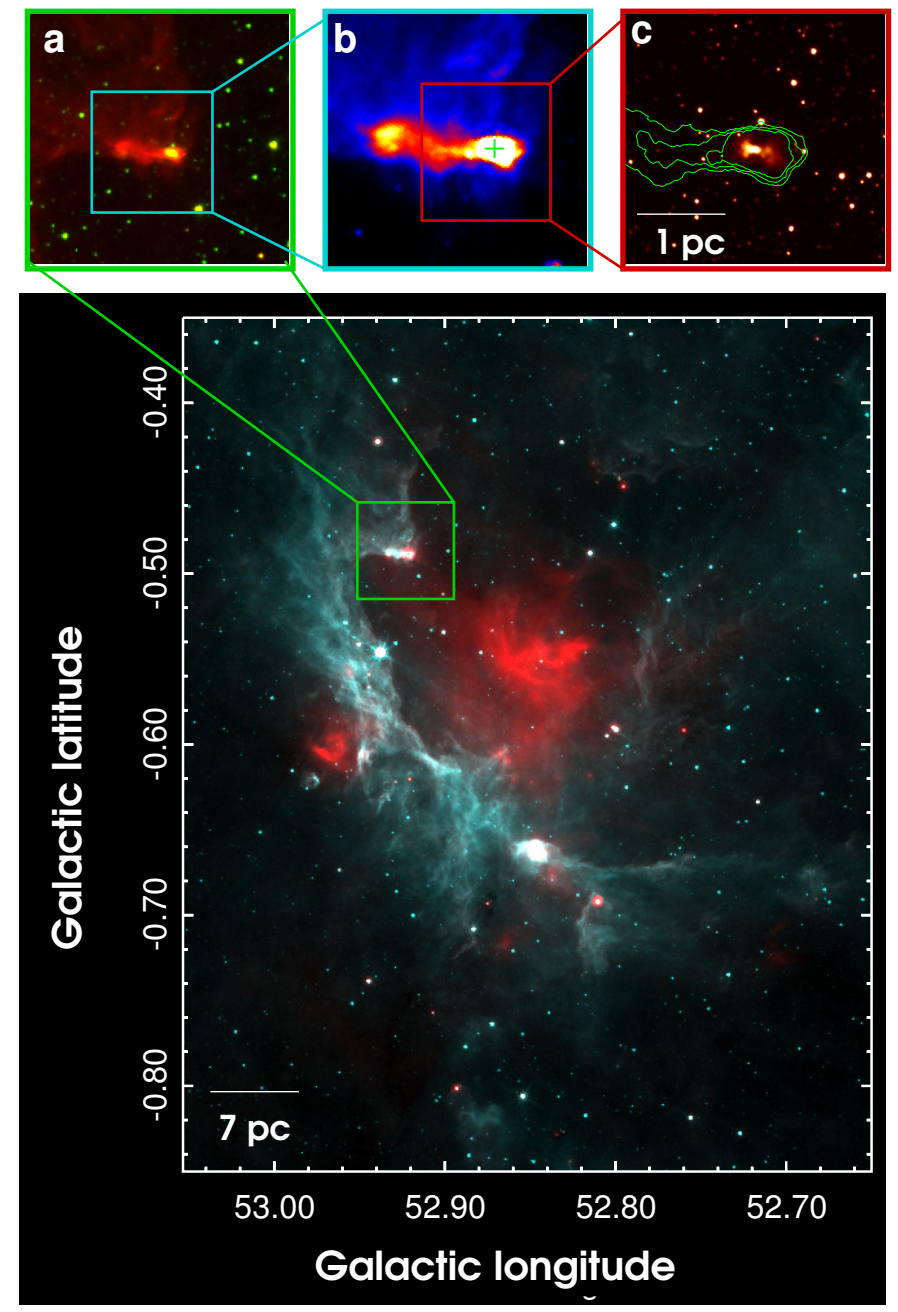

Fig. 1. Spitzer two-color image ( $24 \mu \mathrm{m}$ in red and $8 \mu \mathrm{m}$ in cyan) of bubble G52 (infrared counterpart of the H II region G052.9-00.6). Panel a: Magnified view of the pillar-like feature with $4.5 \mu \mathrm{m}$ in green, and $8 \mu \mathrm{m}$ in red. Both scales run from 20 to $150 \mathrm{MJy}$ beam $^{-1}$. Panel $b$ : Magnified view of a jet-like structure seen at $8 \mu \mathrm{m}$. The cross represents the position of MSXG52. Panel $c$ : Magnified view of the proto-star candidate seen at $K_{\mathrm{s}}$ band extracted from the UKIDSS survey. The green contours represent the $8 \mu \mathrm{m}$ emission levels at 80,100 , and $140 \mathrm{MJy} / \mathrm{beam}$.

a hourglass-like morphology with its symmetry axis perfectly aligned with the jet-like feature seen at $8 \mu \mathrm{m}$. This bipolar cone-like shape morphology suggests the presence of cavities cleared in the circumstellar material. These kind of cavities can be originated by the action of a wide-angle wind arising from a proto-star at its latest stages of evolution or a young star that has recently reached the ZAMS (Weigelt et al. 2006), or by a precessing jet that cleared the circumstellar medium (Kraus et al. 2006). The cone-nebulosity that points toward the jet-like feature appears brighter and more collimated than the other one, which probably, given its location, is evolving toward a less dense medium. This would explain the absence of a jet-like feature at $8 \mu \mathrm{m}$ toward lower Galactic longitudes.

Motivated by the interesting morphology exhibited by MSXG52 in the near- and mid-infrared bands, we carried out molecular lines, and radio continuum observations in order to unveil the nature of this source in the context of the currently accepted massive star formation evolutionary models. In this paper, we report the characterization of the molecular and the ionized 

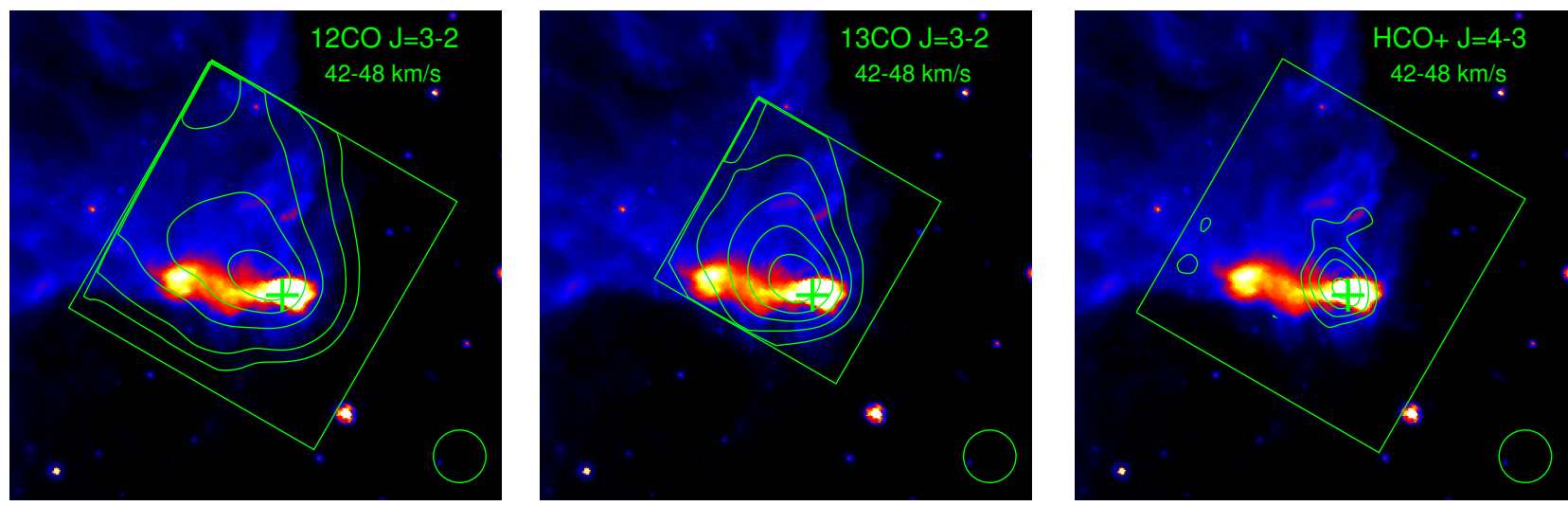

Fig. 2. Averaged velocity maps of ${ }^{12} \mathrm{CO} J=3-2,{ }^{13} \mathrm{CO} J=3-2$, and $\mathrm{HCO}^{+} J=4-3$ emissions between 42 and $48 \mathrm{~km} \mathrm{~s}^{-1}$. The boxes represent the region mapped with the ASTE telescope. The cross indicates the position of the MSXG52. Contours levels are at 1, 2, 3, 5, and 7 K for ${ }^{12} \mathrm{CO} J=3-2$, at $1,1.5,2,2.5,3,3.5 \mathrm{~K}$ for ${ }^{13} \mathrm{CO} J=3-2$, and at $0.4,0.6,0.9$, and $1.2 \mathrm{~K}$ for $\mathrm{HCO}^{+} J=4-3$. Color scale runs from 20 to $150 \mathrm{MJy}^{\text {beam }^{-1}}$.

gas related to MSXG52, based on radio continuum observations at $6 \mathrm{GHz}$ carried out with Karl Jansky Very Large Array (JVLA, USA) and on molecular line data obtained using Atacama Submillimeter Telescope Experiment (ASTE, Chile).

\section{Observations and data reduction}

\subsection{Molecular observations}

The molecular line observations were carried out on $\mathrm{Au}$ gust 27 and 28, 2015 with the $10 \mathrm{~m}$ Atacama Submillimeter Telescope Experiment (ASTE; Ezawa et al. 2004). We used the CATS345 GHz band receiver, which is a side-band separating SIS receiver remotely tunable in the LO frequency range of 324-372 GHz. We simultaneously observed ${ }^{12} \mathrm{CO} J=3-2$ at $345.796 \mathrm{GHz}$ and $\mathrm{HCO}^{+} J=4-3$ at $356.734 \mathrm{GHz}$, mapping a region of $2^{\prime} \times 2^{\prime}$ centered at the position of MSXG52. We also observed ${ }^{13} \mathrm{CO} J=3-2$ at $330.588 \mathrm{GHz}$ and $\mathrm{CS} J=7-6$ at $342.883 \mathrm{GHz}$ toward a region of $1.5 \times 1$. .5 with the same center. The mapping grid spacing was $10^{\prime \prime}$ in both cases, and the integration time was $55 \mathrm{~s}\left({ }^{12} \mathrm{CO}\right.$ and $\left.\mathrm{HCO}^{+}\right)$and $95 \mathrm{~s}\left({ }^{13} \mathrm{CO}\right.$ and $\mathrm{CS}$ ) per pointing. We also performed a single pointing of $\mathrm{C}^{18} \mathrm{O} J=3-2$ at $329.330 \mathrm{GHz}$ toward the same center with an integration time of $19 \mathrm{~min}$. All the observations were performed in position-switching mode. We used the XF digital spectrometer with a bandwidth and spectral resolution set to $128 \mathrm{MHz}$ and $125 \mathrm{kHz}$, respectively. The velocity resolution was $0.11 \mathrm{~km} \mathrm{~s}^{-1}$ and the half-power beam-width $(H P B W)$ was about $22^{\prime \prime}$ for all observed molecular lines. The system temperature varied from $T_{\text {sys }}=150$ to $200 \mathrm{~K}$. The main beam efficiency was $\eta_{\mathrm{mb}} \sim 0.65$.

The data were reduced with NEWSTAR ${ }^{1}$ and the spectra processed using the XSpec software package ${ }^{2}$. All the spectra were Hanning-smoothed to improve the signal-to-noise ratio. The baseline fitting was carried out using second order polynomials for the ${ }^{12} \mathrm{CO},{ }^{13} \mathrm{CO}$, and $\mathrm{C}^{18} \mathrm{O}$ transitions and third-order polynomials for the $\mathrm{HCO}^{+}$transition. The resulting rms noise of the observations was about $0.15 \mathrm{~K}$ for ${ }^{13} \mathrm{CO} J=3-2$ and $\mathrm{CS} J=7-6,0.07 \mathrm{~K}$ for ${ }^{12} \mathrm{CO} J=3-2$ and $\mathrm{HCO}^{+} J=4-3$ and $0.04 \mathrm{~K}$ for $\mathrm{C}^{18} \mathrm{O} J=3-2$ transitions.

\footnotetext{
1 Reduction software based on AIPS developed at NRAO, ex- tended to treat single dish data with a graphical user interface (GUI).

$2 \mathrm{XSpec}$ is a spectral line reduction package for astronomy which has been developed by Per Bergman at Onsala Space Observatory.
}

\subsection{Radio continuum observations}

The radio continuum observations toward MSXG52 were performed in a single pointing with the Karl G. Jansky Very Large Array (JVLA) in its C configuration, on February 11, 2016 (project ID:16A-058) for a total of $40 \mathrm{~min}$ on-source integration time. We used the wide-band $4-8 \mathrm{GHz}$ receiver system centered at 5.5 and $6.5 \mathrm{GHz}$, which consists in 16 spectral windows with a bandwidth of $128 \mathrm{MHz}$ each, spread into 64 channels. Data processing was carried out using the CASA and Miriad software packages, following standard procedures. The source $\mathrm{J} 1331+305$ was used for primary flux density and bandpass calibration, while phases were calibrated with J1931-2243. We reconstructed an image centered at $6 \mathrm{GHz}$ with a bandwidth of $2 \mathrm{GHz}$ using the task MAXEN in MIRIAD, which performs a maximum entropy deconvolution algorithm on a cube. The resulting synthesized beam has a size of 4 ". $8 \times 4$ ".'1, and the rms noise of the final map is $40 \mu \mathrm{Jy}_{\text {beam }^{-1}}$.

\section{Results and discussion}

\subsection{Molecular gas and dust}

Figure 2 shows the averaged velocity maps of ${ }^{12} \mathrm{CO} J=3-2$, ${ }^{13} \mathrm{CO} J=3-2$, and $\mathrm{HCO}^{+} J=4-3$ emissions between 42 and $48 \mathrm{~km} \mathrm{~s}^{-1}$. The ${ }^{12} \mathrm{CO} J=3-2$ and ${ }^{13} \mathrm{CO} J=3-2$ emission distributions perfectly match the pillar structure with a conspicuous molecular clump placed toward the head of this structure. MSXG52 (indicated as a green cross in the figure) appears placed onto the border of this clump that faces the H II region. The $\mathrm{HCO}^{+} J=4-3$ emission appears concentrated toward the head of the pillar in coincidence with the position of the Red MSX source. Figure 3 shows the ${ }^{12} \mathrm{CO} J=3-2,{ }^{13} \mathrm{CO} J=3-2$, $\mathrm{HCO}^{+} J=4-3$, and $\mathrm{C}^{18} \mathrm{O} J=3-2$ spectra taken toward the position of MSXG52 and Table 1 shows the derived parameters from Gaussian fits.

Column density toward the position of the MSXG52 was derived from the ${ }^{13} \mathrm{CO}$ and $\mathrm{C}^{18} \mathrm{O}$ data by assuming a filled beam and a uniform excitation temperature, common to both tracers (local thermodynamic equilibrium assumption), within the beam. We first derive the ${ }^{13} \mathrm{CO}$ opacity, $\tau_{13}$, based on the following equation:

$\frac{T_{\mathrm{mb}}\left({ }^{13} \mathrm{CO}\right)}{T_{\mathrm{mb}}\left(\mathrm{C}^{18} \mathrm{O}\right)}=\frac{1-\exp \left(-\tau_{13}\right)}{1-\exp \left(-\tau_{18}\right)}$ 

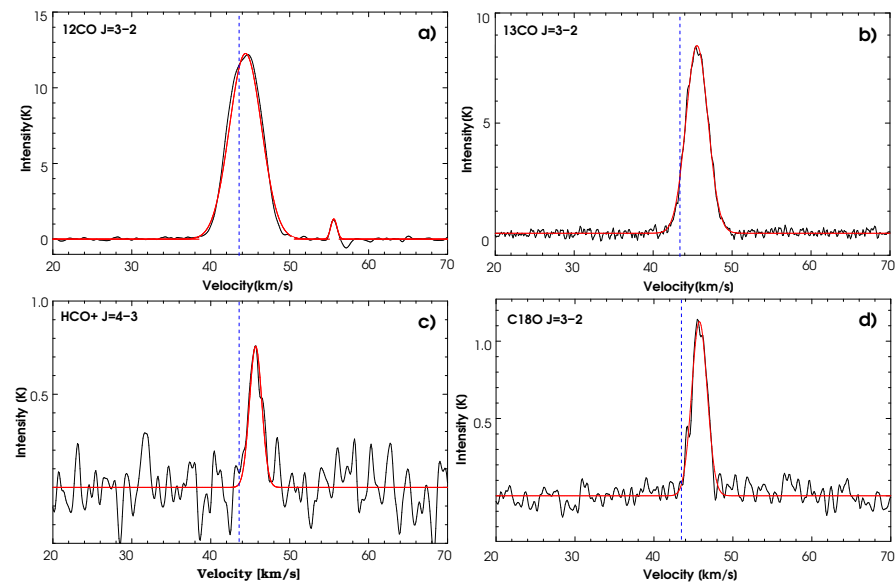

Fig. 3. ${ }^{12} \mathrm{CO} J=3-2 a$ ), ${ }^{13} \mathrm{CO} J=3-2 b$ ), $\mathrm{HCO}^{+} J=4-3 c$ ), and $\left.\mathrm{C}^{18} \mathrm{O} J=3-2 d\right)$ spectra obtained toward the position of the MSXG52. The dashed line indicates the radio recombination line related to G52. The Gaussian used to fit the spectra is shown in red.

where we consider $T_{\mathrm{mb}}$ at the position of MSXG52. Assuming $\tau_{13}=7.4 \tau_{18}$, based on the abundance ratio $\left[{ }^{13} \mathrm{C}\right]\left[{ }^{16} \mathrm{O}\right] /\left[{ }^{12} \mathrm{C}\right]\left[{ }^{18} \mathrm{O}\right]=7.4$ estimated at a galacto-centric radius of $D_{\mathrm{GC}}=5.5 \mathrm{kpc}$ (Wilson \& Rood 1994), we derive a $\tau_{13} \sim 0.2$, and a $\tau_{18} \sim 0.02$, which shows that both transitions are optically thin toward the position of the MSX source. The excitation temperature can then be derived from the equation of radiative transfer applied to the ${ }^{13} \mathrm{CO} J=3-2$ transition,

$T_{\mathrm{mb}}\left({ }^{13} \mathrm{CO}\right)=\frac{h v}{k}\left(\frac{1}{\exp \left(\frac{h v}{k T_{\mathrm{ex}}}\right)-1}-\frac{1}{\exp \left(\frac{h v}{k T_{\mathrm{BG}}}\right)-1}\right) \times\left(1-\exp \left(-\tau_{13}\right)\right)$,

where $\mathrm{h} v / k=15.87 \mathrm{~K}$ and $T_{\mathrm{BG}}=2.7 \mathrm{~K}$. We obtain a $T_{\mathrm{ex}}$ of about $52 \mathrm{~K}$. Given $T_{\mathrm{ex}}$ and $\tau_{13}$, the column density of the ${ }^{13} \mathrm{CO}$ can be derived from (e.g., Buckle et al. 2010),

$N\left({ }^{13} \mathrm{CO}\right)=8.28 \times 10^{13} \mathrm{e}^{\frac{h v}{k T_{\mathrm{ex}}}} \frac{T_{\mathrm{ex}}+0.88}{1-\exp \left(\frac{-h v}{k T_{\mathrm{ex}}}\right)} \int \tau_{13} \mathrm{~d} v$.

Taking into account that ${ }^{13} \mathrm{CO} J=3-2$ transition is optically thin, the following approximation can be used,

$\int \tau \mathrm{d} v=\frac{1}{J\left(T_{\mathrm{ex}}\right)-J\left(T_{\mathrm{BG}}\right)} \int T_{\mathrm{mb}} \mathrm{d} v$,

with

$J(T)=\frac{h v / k}{\exp \left(\frac{h v}{k T}\right)-1}$,

where $\int T_{\mathrm{mb}} \mathrm{d} v \sim 28 \mathrm{~K} \mathrm{~km} \mathrm{~s}^{-1}$. From the estimated $N\left({ }^{13} \mathrm{CO}\right) \sim$ $2.1 \times 10^{15} \mathrm{~cm}^{-2}$, and assuming the $\left[\mathrm{H}_{2}\right] /\left[{ }^{13} \mathrm{CO}\right]$ ratio of $77 \times 10^{4}$ (Wilson \& Rood 1994), we derive a $\mathrm{H}_{2}$ column density of $\sim 1.6 \times$ $10^{21} \mathrm{~cm}^{-2}$

In order to achieve a characterization of the ambient conditions that includes the more external layers of gas toward this region, we derive the excitation temperature and the column density using the ${ }^{12} \mathrm{CO} J=3-2$ and ${ }^{13} \mathrm{CO} J=3-2$ transitions. Based on the ratio $T_{\mathrm{mb}}\left({ }^{12} \mathrm{CO}\right) / T_{\mathrm{mb}}\left({ }^{13} \mathrm{CO}\right)$ of about 1.5 , we estimate opacities of about 55 and 1 for the ${ }^{12} \mathrm{CO} J=3-2$ and ${ }^{13} \mathrm{CO} J=3-2$ lines, respectively, and derive a $T_{\mathrm{ex}} \sim 20 \mathrm{~K}$.
Table 1. Parameters derived from a Gaussian fitting of the spectra shown in Fig. 3.

\begin{tabular}{cccc}
\hline \hline Transition & $T_{\mathrm{mb}}[\mathrm{K}]$ & $V_{\mathrm{LSR}}\left[\mathrm{km} \mathrm{s}^{-1}\right]$ & $\Delta v\left[\mathrm{~km} \mathrm{~s}^{-1}\right]$ \\
\hline${ }^{12} \mathrm{CO} J=3-2$ & $12.3 \pm 0.6$ & $44.6 \pm 0.8$ & $4.5 \pm 0.6$ \\
& $1.3 \pm 0.2$ & $55.6 \pm 0.4$ & $0.9 \pm 0.4$ \\
\hline${ }^{13} \mathrm{CO} J=3-2$ & $8.1 \pm 0.5$ & $45.4 \pm 0.5$ & $3.4 \pm 0.4$ \\
\hline $\mathrm{C}^{18} \mathrm{O} J=3-2$ & $1.2 \pm 0.3$ & $45.7 \pm 0.6$ & $3.1 \pm 0.5$ \\
\hline $\mathrm{HCO}^{+} J=4-3$ & $0.7 \pm 0.2$ & $45.5 \pm 0.4$ & $2.3 \pm 0.6$ \\
\hline
\end{tabular}

Using Eq. (3) and assuming a canonical $\left[{ }^{12} \mathrm{CO}\right] /\left[{ }^{13} \mathrm{CO}\right]$ isotope abundance ratio of 50 , we derive a $N\left(\mathrm{H}_{2}\right)$ of about $5 \times 10^{21} \mathrm{~cm}^{-2}$. Thus, comparing these results with those obtained for ${ }^{13} \mathrm{CO} J=$ 3-2 and $\mathrm{C}^{18} \mathrm{O} J=3-2$ transitions, under LTE conditions ( $T_{\text {ex }} \sim T_{\text {kin }}$, we suggest kinetic temperature and $\mathrm{H}_{2}$ column density gradients across the molecular clump, with the hotter and less dense gas in the inner region. This scenario is in agreement with the presence of a proto-star at its latest stages of evolution embedded in the molecular clump.

On the other hand, from the far-infrared and submillimeter continuum emission of the dust, we estimate a temperature of the region that can be compared with those obtained above. Under the assumption that the dust radiates as a graybody characterized by a single temperature $T_{\mathrm{d}}$ and that the emission is optically thin, the flux at a frequency $v$ is $S_{v} \propto N_{\mathrm{d}} k_{v} B_{v}\left(T_{\mathrm{d}}\right)$ (Anderson et al. 2012), where $B_{v}\left(T_{\mathrm{d}}\right)=2 h v^{3} / c^{2}\left[\exp \left(h v /\left(k_{\mathrm{B}} T_{\mathrm{d}}\right)\right)-1\right]^{-1}$ is the Planck function, $k_{v}=k_{v 0}\left(v / v_{0}\right)^{\beta}$ is the dust opacity (with $\beta=2$, as observed in a large sample of galactic H II regions) and $N_{\mathrm{d}}$ is the dust column density. The above equation can be written in terms of the $\mathrm{H}_{2}$ column density as

$S_{v}=\mu m_{\mathrm{H}} N\left(\mathrm{H}_{2}\right) k_{v 0}\left(v / v_{0}\right)^{\beta} B_{v}\left(T_{\mathrm{d}}\right)$.

For a frequency $v_{0}=1 \mathrm{THz}$ and a gas-to-dust ratio of 100, we take $k_{v 0}=0.1 \mathrm{~cm}^{2} \mathrm{~g}^{-1}$. We adopt a mean molecular weight $\mu=2.8$, corresponding to a relative helium abundance of $10 \%$. We use Herschel observations to derive the fluxes at different bands and fit the spectral energy distribution (SED) of the dust emission with Eq. (6). Observations \#1342231341 and \#1342231342 cover the field around G52 and we used level 2.5 data, which combine both observations in a single set of images. We fitted the SED using Herschel-PACS $160 \mu \mathrm{m}$ and Herschel-SPIRES 250, 350 and $500 \mu \mathrm{m}$ bands. We estimated the flux of the more intense pixel at the four Herschel images, which corresponds to the position of MSXG52, and subtracted the contribution of the background emission by measuring the mean flux from a circular region centered at $l \sim 52.95^{\circ}, b \sim-0.48^{\circ}$ with a radius of $35^{\prime \prime}$. The background-subtracted peak fluxes are: 6349, 1644, 473 and $111 \mathrm{MJy} \mathrm{sr}^{-1}$ at 160, 250, 350 and $500 \mu \mathrm{m}$, respectively. Flux uncertainties of the Herschel-PACS and -SPIRES photometers are estimated in $~ 5 \%$ (Balog et al. 2014 and Bendo et al. 2013, respectively). We fitted the data using Eq. (6) leaving $N\left(\mathrm{H}_{2}\right)$ and $T_{\mathrm{d}}$ as free parameters. The best-fit dust temperature was $T_{\mathrm{d}}=48.1 \pm 9.7 \mathrm{~K}$ and $\mathrm{H}_{2}$ column density $N\left(\mathrm{H}_{2}\right)=(2.2 \pm 1.0) \times 10^{21} \mathrm{~cm}^{-2}$, with quoted errors corresponding to the $95 \%$ confidence range. These results support the estimations derived from the molecular observations toward the gas in the inner region. In Fig. 4 we plot the fluxes and the best-fit graybody model. 


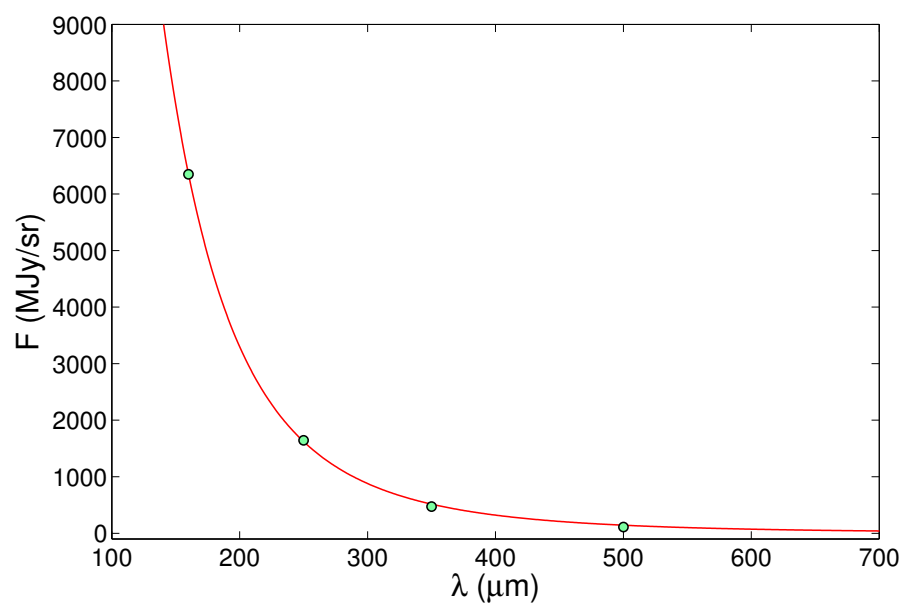

Fig. 4. Far-infrared and submillimeter continuum fluxes of MSXG52 in the Herschel 160, 250, 350 and $500 \mu \mathrm{m}$ bands. The red line is the best-fit graybody model (Eq. (6)) corresponding to a dust temperature of $\sim 48 \mathrm{~K}$ and $\mathrm{H}_{2}$ column density of $\sim 2 \times 10^{21} \mathrm{~cm}^{-2}$.

The source MSXG52 exhibits in the $8 \mu \mathrm{m}$ and $K_{\mathrm{s}}$ band images a morphology very suggestive of outflow activity in the region. A detailed inspection of the whole ${ }^{12} \mathrm{CO} J=3-2$ data cube revealed the presence of spectral wings in some ${ }^{12} \mathrm{CO} J=3-2$ spectra (shown in Fig. 5), likely associated with molecular outflows. Figure 5 shows Spitzer-IRAC image at $8 \mu \mathrm{m}$ of MSXG52. The ${ }^{12} \mathrm{CO} J=3-2$ emission averaged from 36 to $42 \mathrm{~km} \mathrm{~s}^{-1}$ (blue lobe), and from 48 to $51 \mathrm{~km} \mathrm{~s}^{-1}$ (red lobe) are shown in the figure. The feature B1 related to the blue-shifted emission appears located in projection onto the jet-like structure as seen at $8 \mu \mathrm{m}$. The spectrum obtained toward the center of this lobe exhibits a blue wing highlighted in the figure. The red-shifted emission exhibits a more complex morphology with two features $\mathrm{R} 1$, and $\mathrm{R} 2$ that are misaligned with respect to the axis of the jet-like structure. In particular, the spectrum obtained toward the center of source R2 exhibits a velocity component centered at $49 \mathrm{~km} \mathrm{~s}^{-1}$, which could be due to a red-shifted bullet of molecular gas. The velocity component centered at $55 \mathrm{~km} \mathrm{~s}^{-1}$, which is observed in the three spectra, corresponds to molecular gas associated with the pillar structure.

To roughly estimate the outflow mass, following Bertsch et al. (1993), we calculated the $\mathrm{H}_{2}$ column density from

$N\left(\mathrm{H}_{2}\right)=2.0 \times 10^{20} \frac{W\left({ }^{12} \mathrm{CO}\right)}{\mathrm{K} \mathrm{km} \mathrm{s}^{-1}}\left(\mathrm{~cm}^{-2}\right)$,

where $W\left({ }^{12} \mathrm{CO}\right)$ is the ${ }^{12} \mathrm{CO} J=3-2$ integrated intensity along the intervals mentioned above. Then, the mass was derived from

$M=\mu m_{\mathrm{H}} D^{2} \Omega \sum_{i} N_{\mathrm{i}}\left(\mathrm{H}_{2}\right)$,

where $\Omega$ is the solid angle subtended by the beam size, $m_{\mathrm{H}}$ is the hydrogen mass, $\mu=2.8$ corresponds to a relative helium abundance of $10 \%$, and $D$ is the distance. We summed over all beam positions belonging to the lobes, which yields the mass for the red- and blue-shifted outflows: $M_{\text {red }} \sim 6 M_{\odot}$ and $M_{\text {blue }} \sim$ $5 M_{\odot}$, in agreement with typical values for massive molecular outflows (Wu et al. 2004).

From a simple inspection of the calibrated near-infrared (NIR) spectrum in the $\mathrm{H}+\mathrm{K}$ bands toward MSXG52 (Cooper et al. 2013), kindly provided by Lumsden S., it can be appreciated a prominent $\mathrm{Br} \gamma$ emission line, and no evidence of

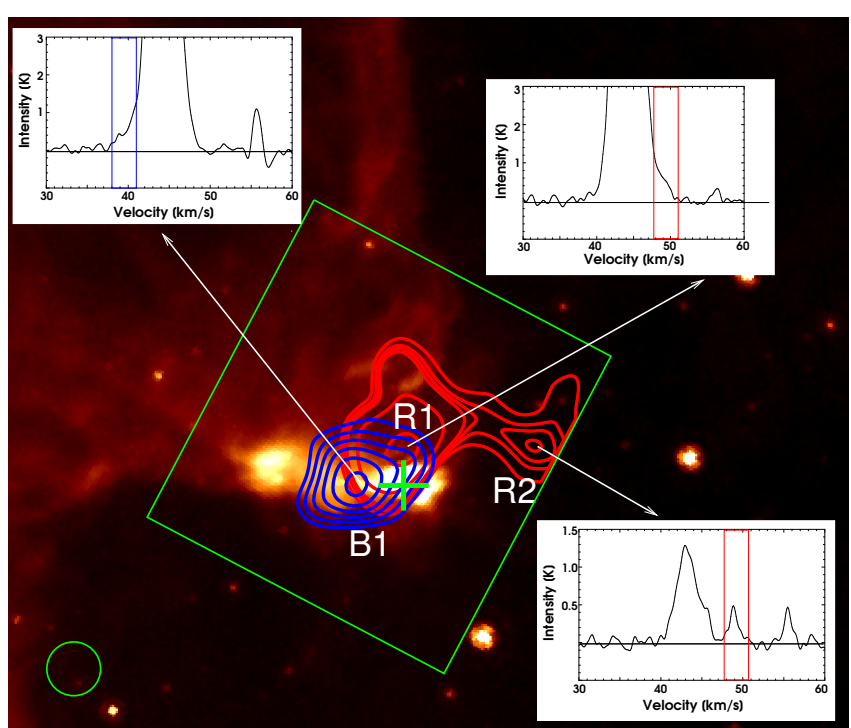

Fig. 5. Spitzer-IRAC image at $8 \mu \mathrm{m}$ of MSXG52. The blue and red contours represent the ${ }^{12} \mathrm{CO} J=3-2$ emission averaged from 36 to $42 \mathrm{~km} \mathrm{~s}^{-1}$ (blue lobe), and from 48 to $51 \mathrm{~km} \mathrm{~s}^{-1}$ (red lobe), respectively. The blue contours are at $0.10,0.12,0.14,0.16$, and $0.18 \mathrm{~K}$ and the red ones are at $0.10,0.12,0.13,0.14,0.20,0.30 \mathrm{~K}$. We show the spectra toward the center of features B1, R1, and R2. The green cross indicates the location of MSXG52. The green box shows the area mapped with ASTE at this transition. The green circle represents the beam of the molecular observations.

$\mathrm{H}_{2}$ 1-0 S(1) and [FeII] lines. This Bry emission might arise from ionized stellar wind generated in an evolved massive proto-star (Bik et al. 2006; Kraus et al. 2008) or from a compact H II region in photoionization equilibrium (Krolik \& Smith 1981). Additionally, the non-detection of $\mathrm{H}_{2} \quad 1-0 \mathrm{~S}(1)$ and [FeII] lines would suggest the absence of collimated jets shocking the inner regions of a YSO envelopment (Reipurth et al. 2000; Bally et al. 2007). Thus, the lack of evidence of collimated jets would suggest that the outflow activity in MSXG52 would be related to a wide-angle ionized stellar wind. Weigelt et al. (2006) suggest that a wide-angle stellar wind still plays an important role in driving molecular outflows.

\subsection{The ionized gas}

Figure 6a shows a two-color image with the $8 \mu \mathrm{m}$ emission in red and the radio continuum emission at $6 \mathrm{GHz}$ in blue. We note that the diffuse radio continuum emission perfectly matches the north border of the pillar-like structure as seen at $8 \mu \mathrm{m}$. This emission seems to arise from the ionized gas related to the large $\mathrm{H}$ II region that has been stalled against the pillar, illuminating it. This is in agreement with the assumption that the exciting star(s) of the large $\mathrm{H}$ II region is(are) located in the bulk of emission at $24 \mu \mathrm{m}$ (see Fig. 1). By the other hand, there are two conspicuous compact radio sources lying along the southern border of the pillar. One of them, named C1, coincides in position with MSXG52. The other source, $\mathrm{C} 2$, is seen in projection onto the tail of the jet-like feature. As can be appreciated from Fig. 7, source C1 shows a slightly elongated morphology at $6 \mathrm{GHz}$ with respect to source C2. In particular, C1 embraces perfectly the UKIDSS source UGPS J193054.62+172842.0, which is the near-infrared counterpart of MSXG52 at the $K_{\mathrm{s}}$ band. At this band, it can be noticed the young star at the center of the hourglass-like nebula, 

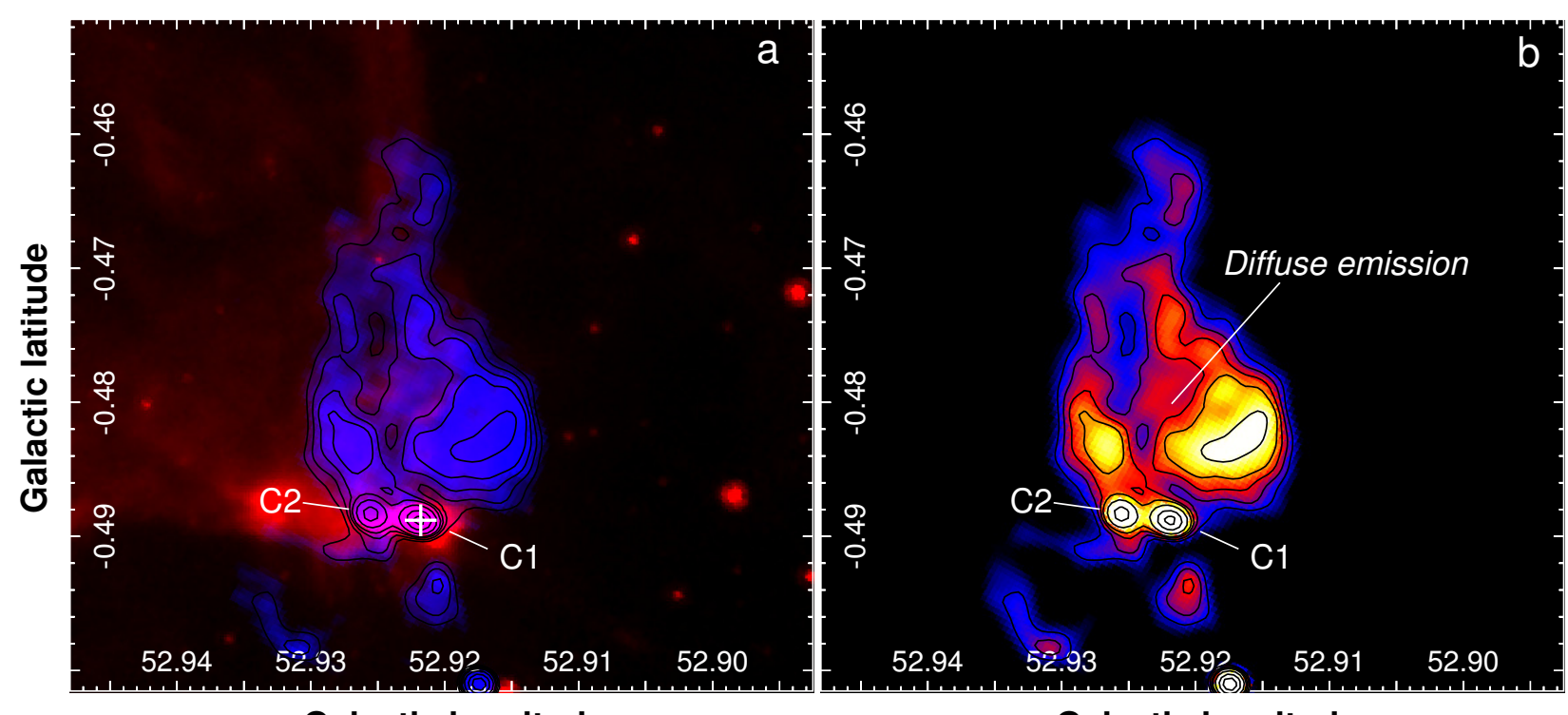

Galactic longitude

Galactic longitude

Fig. 6. Panel $a$ : two-color image with $8 \mu \mathrm{m}$ in red and $6 \mathrm{GHz}$ in blue. The cross indicates the position of MSXG52. Panel $b$ : radio continuum emission at $6 \mathrm{GHz}$. The scale goes from 0.08 to $0.3 \mathrm{mJy}_{\text {beam }}{ }^{-1}$. Levels are at $0.12,0.14,0.16,0.19,0.25$, and $0.35 \mathrm{mJy} \mathrm{beam}^{-1}$. C1 and C2 show the position of the two compact radio sources mentioned in the text.

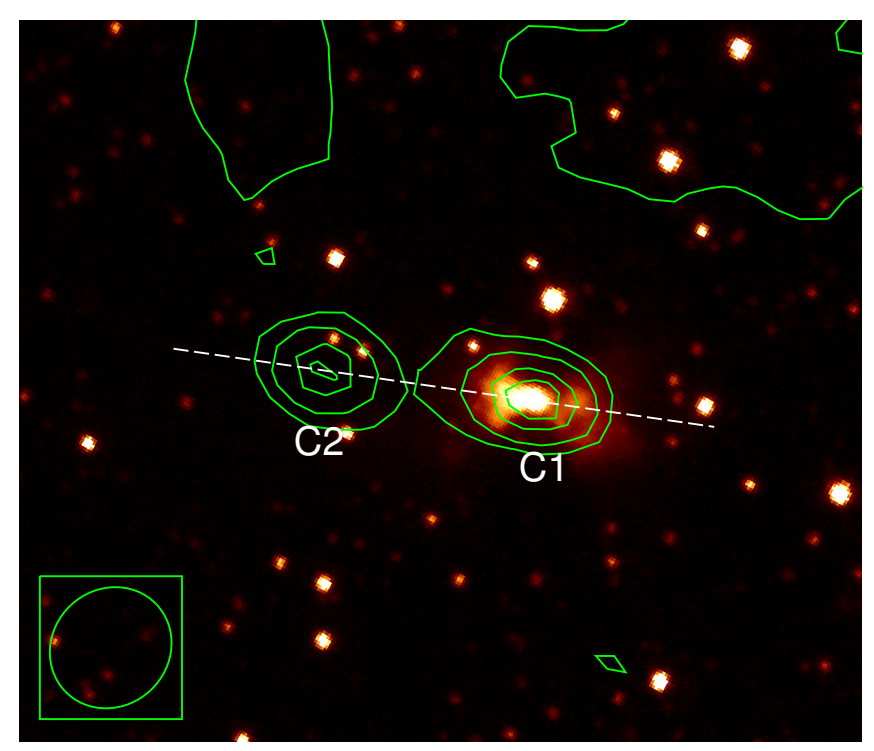

Fig. 7. NIR emission at the $K_{\mathrm{s}}$ band as extracted from the UKIDSS Survey. The green contours represent the radio continuum emission at $6 \mathrm{GHz}$ at $0.2,0.3,0.4$, and $0.5 \mathrm{mJy}^{\text {beam }^{-1}}$. The beam of the radio continuum observation is shown in the bottom-left corner.

which suggests the presence of cavities cleared in the circumstellar material. Moreover, the young star coincides in position with the peak of the radio emission of $\mathrm{C} 1$. The axis of symmetry connecting both compact radio sources (indicated in Fig. 7) shows a perfect alignment with the axis of symmetry of the hourglasslike nebula and with the jet-like feature seen at $8 \mu \mathrm{m}$, which suggests that these structures are related.

The primary mechanism of the radio continuum emission for MYSOs is thermal free-free emission from ionized gas. This may either be in the form of thermal jets, ionized stellar winds, and/or photo-ionized compact H II regions (e.g., Gibb \& Hoare 2007; Rodríguez et al. 2012). In turn, these compact photo-ionized regions can be divided in hyper- and ultra-compact $\mathrm{H}$ II regions, which at $6 \mathrm{GHz}$ are in the optically thick and optically thin regime, respectively (Kurtz 2005). The estimated radio flux density for $\mathrm{C} 1$ at $6 \mathrm{GHz}$ of about $0.9 \mathrm{mJy}$, is in agreement with all these scenarios (Rodríguez et al. 2012).

In this context, a first clue to the origin of the ionized gas associated with MSXG52 and therefore of the nature of the source, could be provided by a comparison between the $\mathrm{Br} \gamma$ intensity and the radio continuum emission at $6 \mathrm{GHz}$. From the NIR spectrum kindly provided by Lumsden $\mathrm{S}$. (Cooper et al. 2013), we derived a Br $\gamma$ intensity of about $0.13 \times$ $10^{-12} \mathrm{erg} \mathrm{cm}^{-2} \mathrm{~s}^{-1}$. Assuming a ratio $\mathrm{Br} \alpha / \mathrm{Br} \gamma$ of about 2.33 (Wynn-Williams 1984), and a radio continuum emission at the optically thin regime, we estimated the ratio $\mathrm{Br} \alpha / \mathrm{S}(5 \mathrm{GHz})$ of about $0.033 \times 10^{-12} \mathrm{erg} \mathrm{cm}^{-2} \mathrm{~s}^{-1} \mathrm{mJy}^{-1}$, which is in agreement with the predicted value of $0.026 \times 10^{-12} \mathrm{erg} \mathrm{cm}^{-2} \mathrm{~s}^{-1} \mathrm{mJy}^{-1}$ for an optically thin H II region in photoionization equilibrium (Snell \& Bally 1986). This result would rule out the presence of an optically thick envelope surrounding a star arising in a ionized stellar wind (Krolik \& Smith 1981). It is important to mention that considering the radio continuum emission at the optically thick regime (Kurtz 2005), only introduces a factor of about 1.5 in the $\operatorname{Br} \alpha / \mathrm{S}(5 \mathrm{GHz})$ ratio derived above. This result suggests that the radio source $\mathrm{C} 1$ would be the ionized gas of an optically thin UC HII region associated with a young massive star. If this is the case, we can conjecture upon the spectral type of a probable single ZAMS star based on the estimated radio flux density for $\mathrm{C} 1$. The number of photons needed to keep an $\mathrm{H}$ II region ionized, in an optically thin regime, is given by $N_{\mathrm{uv}}=0.76 \times 10^{47} T_{4}^{-0.45} v_{\mathrm{GHZ}}^{0.1} S_{v} D_{\mathrm{kpc}}^{2}$ (Chaisson 1976), where $T_{4}$ is the electron temperature in units of $10^{4} \mathrm{~K}, D_{\mathrm{kpc}}$ is the distance in kilo-parsecs, $v_{\mathrm{GHz}}$ is the frequency in $\mathrm{GHz}$, and $S_{v}$ is the measured total flux density in Jansky. We assumed an electron temperature of $T=10^{4} \mathrm{~K}$ and a distance of $7.2 \mathrm{kpc}$. We derived a total amount of ionized photons in $\mathrm{C} 1$ of about $N_{u v}=(4.2 \pm 2.2) \times 10^{45} \mathrm{ph} \mathrm{s}^{-1}$. Based on Avedisova (1979) and Martins et al. (2005), we conclude that the spectral type of the exciting star of this wimpy UC H II region should be B0.5V. 
On the other hand, the presence of molecular outflows related to MSXG52, together with the slightly elongated morphology of the associated radio continuum emission (source $\mathrm{C} 1$ ), would suggest that the source is transiting an earlier evolutionary stage. If this is the case, given the lack of evidence of collimated jets in the near-infrared spectrum (see Sect. 4.1.1), we could find an explanation to its elongated morphology in the work of Keto (2007), which suggests that at some point during its evolution, an HC H II region exhibits a bipolar morphology that accounts for the presence of wide-angle ionized stellar winds flowing through the poles. The hourglass morphology of the source at the $K_{\mathrm{s}}$ band is in agreement with this scenario. Thus, in the context of the evolutionary models for high-mass stars proposed by Beuther \& Shepherd (2005), we suggest that MSXG52 could be at the evolutionary stage in which the jets are giving way to the wide-angle ionized winds together with a photo-ionized region around the young star. Thus, the jet-like feature seen at $8 \mu \mathrm{m}$ and the hourglass nebula detected at the $K_{\mathrm{s}}$ band would be generated by the action of the UV photons and winds that escape from the polar regions of the young star.

Regarding the nature of the source $\mathrm{C} 2$, its perfect alignment with the axis of symmetry of the radio source $\mathrm{C} 1$, together with the absence of an IR counterpart, would suggest that this radio source is tracing an ionized stellar wind breaking out the central source in an episodic ejection event. However, the possibility that $\mathrm{C} 2$ is related to a companion MYSO can not be ruled out.

\section{Summary and conclusion}

In this paper, we study the molecular and ionized gas in the Red MSX source G052.9221-00.4892. The ${ }^{12} \mathrm{CO} J=3-2$ emission shows the presence of misaligned high-mass molecular outflows. In particular, the blue lobe coincides in position with a jet-like structure, detected at $8 \mu \mathrm{m}$, that arises from the central source. However, from the NIR spectrum, we do not find signatures of collimated jets related to this source. The radio continuum emission at $6 \mathrm{GHz}$ shows a slightly elongated radio compact source in positional coincidence with the Red MSX source. This radio source perfectly matches the hourglass nebula seen at the $K_{\mathrm{S}}$ band. Thus, the presence of molecular outflows related to the Red MSX source G052.9221-00.4892, the slightly elongated morphology of the associated radio continuum emission matching the hourglass-like morphology exhibited by the source at the $K_{\mathrm{s}}$ band, and the lack of evidence of collimated jets, suggest that the source could be transiting the earlier evolutionary stage of $\mathrm{HC} \mathrm{HII}$ region with the presence of a bipolar wideopen angle ionized wind. Keto (2007) and Beuther \& Shepherd (2005) predict an evolutionary stage in the massive stellar formation in which the star reaches the ZAMS and continues with the accretion mainly through the equatorial plane causing an outwards flow of ionized gas and winds emanating from the poles. Our studied source could be observational evidence of this stage. Taking into account that the cataloged HC H II regions are scarce, finding and characterizing sources such as that studied in this work is very important to advance in the understanding of the massive star formation and how the stars build up their masses once they have reached the ZAMS.

On the other hand, according to a comparison between the Br $\gamma$ intensity and the radio flux density at $6 \mathrm{GHz}$, the source would be in a more evolved evolutionary stage of an optically thin UC HII region in photoionization equilibrium. If this is the case, from the estimated radio flux density, we can conjecture upon the spectral type of its exciting star which would be a B0.5V.
Acknowledgements. We acknowledge the anonymous referee for her/his helpful comments and suggestions. We wish to thank to S. Lumsden for kindly providing us with the NIR spectrum of the Red MSX source G052.9221-00.4892. The ASTE project is led by Nobeyama Radio Observatory (NRO), a branch of National Astronomical Observatory of Japan (NAOJ), in collaboration with University of Chile, and Japanese institutes including University of Tokyo, Nagoya University, Osaka Prefecture University, Ibaraki University, Hokkaido University, and the Joetsu University of Education. M.O., S.P., E.G., and A.P. are members of the Carrera del investigador científico of CONICET, Argentina. M.C.P. is a doctoral fellow of CONICET, Argentina. This work was partially supported by grants awarded by CONICET, ANPCYT and UBA (UBACyT) from Argentina. A.P. acknowledges the support from the Varsavsky Foundation. M.R. wishes to acknowledge support from FONDECYT(CHILE) grant No. 1140839. M.O., S.P., and A.P. are grateful to Dr. Takeshi Okuda for the support received during the ASTE observations.

\section{References}

Anderson, L. D., \& Bania, T. M. 2009, ApJ, 690, 706

Anderson, L. D., Zavagno, A., Deharveng, L., et al. 2012, A\&A, 542, A10 Avedisova, V. S. 1979, Sov. Astron., 23, 544

Bally, J., Reipurth, B., \& Davis, C. J. 2007, Protostars and Planets V, 215 Balog, Z., Müller, T., Nielbock, M., et al. 2014, Exp. Astron., 37, 129 Bendo, G. J., Griffin, M. J., Bock, J. J., et al. 2013, MNRAS, 433, 3062 Benjamin, R. A., Churchwell, E., Babler, B. L., et al. 2003, PASP, 115, 953 Bertsch, D. L., Dame, T. M., Fichtel, C. E., et al. 1993, ApJ, 416, 587

Beuther, H., \& Shepherd, D. 2005, in Cores to Clusters: Star Formation with Next Generation Telescopes, eds. M. S. N. Kumar, M. Tafalla, \& P. Caselli, 324, 105

Bik, A., Kaper, L., \& Waters, L. B. F. M. 2006, A\&A, 455, 561

Bonnell, I. A., \& Bate, M. R. 2006, MNRAS, 370, 488

Buckle, J. V., Curtis, E. I., Roberts, J. F., et al. 2010, MNRAS, 401, 204

Carey, S. J., Noriega-Crespo, A., Mizuno, D. R., et al. 2009, PASP, 121, 76

Cesaroni, R., Churchwell, E., Hofner, P., Walmsley, C. M., \& Kurtz, S. 1994, A\&A, 288, 903

Chaisson, E. J. 1976, in Frontiers of Astrophysics, ed. E. H. Avrett, 259

Cooper, H. D. B., Lumsden, S. L., Oudmaijer, R. D., et al. 2013, MNRAS, 430, 1125

Ezawa, H., Kawabe, R., Kohno, K., \& Yamamoto, S. 2004, in Ground-based Telescopes, ed. J. M. Oschmann, Jr., Proc. SPIE, 5489, 763

Garay, G., \& Lizano, S. 1999, PASP, 111, 1049

Gibb, A. G., \& Hoare, M. G. 2007, MNRAS, 380, 246

Hoare, M. G., Purcell, C. R., Churchwell, E. B., et al. 2012, PASP, 124, 939

Keto, E. 2007, ApJ, 666, 976

Keto, E., \& Wood, K. 2006, ApJ, 637, 850

Kraus, S., Balega, Y., Elitzur, M., et al. 2006, A\&A, 455, 521

Kraus, S., Hofmann, K.-H., Benisty, M., et al. 2008, A\&A, 489, 1157

Krolik, J. H., \& Smith, H. A. 1981, ApJ, 249, 628

Kuiper, R., Klahr, H., Beuther, H., \& Henning, T. 2011, ApJ, 732, 20

Kurtz, S. 2005, in Massive Star Birth: A Crossroads of Astrophysics, eds.

R. Cesaroni, M. Felli, E. Churchwell, \& M. Walmsley, IAU Symp., 227, 111 Lockman, F. J. 1989, ApJS, 71, 469

Lucas, P. W., Hoare, M. G., Longmore, A., et al. 2008, MNRAS, 391, 136

Lumsden, S. L., Hoare, M. G., Urquhart, J. S., et al. 2013, ApJS, 208, 11

Martins, F., Schaerer, D., \& Hillier, D. J. 2005, A\&A, 436, 1049

Qiu, K., Zhang, Q., Megeath, S. T., et al. 2008, ApJ, 685, 1005

Reipurth, B., Yu, K. C., Heathcote, S., Bally, J., \& Rodríguez, L. F. 2000, AJ, 120,1449

Rodríguez, L. F., González, R. F., Montes, G., et al. 2012, ApJ, 755, 152

Simpson, R. J., Povich, M. S., Kendrew, S., et al. 2012, MNRAS, 424, 2442

Snell, R. L., \& Bally, J. 1986, ApJ, 303, 683

Tan, J. C., \& McKee, C. F. 2002, in Hot Star Workshop III: The Earliest Phases of Massive Star Birth, ed. P. Crowther, ASP Conf. Ser., 267, 267

Tan, J. C., Beltrán, M. T., Caselli, P., et al. 2014, Protostars and Planets VI, 149 van den Ancker, M. E., Tielens, A. G. G. M., \& Wesselius, P. R. 2000, A\&A, 358, 1035

Warren, S. J., Hambly, N. C., Dye, S., et al. 2007, MNRAS, 375, 213

Weigelt, G., Beuther, H., Hofmann, K.-H., et al. 2006, A\&A, 447, 655

Wilson, T. L., \& Rood, R. 1994, ARA\&A, 32, 191

Wood, D. O. S., \& Churchwell, E. 1989, ApJS, 69, 831

Wu, Y., Wei, Y., Zhao, M., et al. 2004, A\&A, 426, 503

Wynn-Williams, C. G. 1984, in Galactic and Extragalactic Infrared Spectroscopy, eds. M. F. Kessler, \& J. P. Phillips, Astrophys. Space Sci. Lib., 108, 133

Zhang, Y., Tan, J. C., \& Hosokawa, T. 2014, ApJ, 788, 166

Zinnecker, H., \& Yorke, H. W. 2007, ARA\&A, 45, 481 